\title{
LOS SERVICIOS PÚBLICOS Y LA MODERNIZACIÓN DE LA CIUDAD (SIGLOS XIX Y XX)
}

\author{
PRESENTATION: \\ PUBLIC SERVICES AND THE MODERNIZATION OF THE CITY (19th AND \\ 20th CENTURIES) \\ Mercedes Fernández-Paradas*1, Juan Manuel Matés-Barco ${ }^{2}$ \\ ${ }^{1}$ Universidad de Málaga, España \\ ${ }^{2}$ Universidad de Jaén, España
}

* Correspondencia a: Mercedes Fernández-Paradas. Universidad de Málaga. Facultad de Filosofía y Letras. Departamento de Historia Moderna y Contemporánea. Campus Universitario de Teatinos, s/n. Málaga 29071 - paradas@uma.es https://orcid.org/0000-0002-3049-0907

Cómo citar: Fernández-Paradas, Mercedes; Matés-Barco, Juan Manuel (2019). «Presentación: Los servicios públicos y la modernización de la ciudad (siglos XIx y Xx)»; Historia Contemporánea, 59, 11-20. (https://doi.org/10.1387/hc.20344).

ISSN 1130-2402 - eISSN 2340-0277 / (C) 2019 UPV/EHU 
El primer tercio del siglo Xx contempla el crecimiento de numerosas ciudades y poblaciones españolas, que obliga a sus ayuntamientos a buscar fórmulas para desarrollar un proceso de modernización urbanística. Derrumbe de murallas, ensanches, transportes colectivos, red de abastecimiento de agua y alcantarillado, nuevas carreteras, construcción de mercados, escuelas, centros de atención sanitaria, etc., son algunas de las tareas a las que destinaban gran parte de sus presupuestos. Las concentraciones urbanas crecieron de forma espectacular, provocando una serie de ventajas derivadas de la urbanización que se pueden compendiar en la mejora del nivel y la calidad de vida ${ }^{1}$.

La población española seguía siendo predominantemente rural, sobre todo si se considera como urbana a la residente en las capitales de provincia y en localidades cuyo casco urbano supere los 5.000 habitantes. Si el coeficiente de urbanización en 1900 era del 31,2 por 100; treinta años más tarde esta cifra había crecido y se situaba en el 39,4 por 100. Si bien las áreas metropolitanas de Madrid y Barcelona pronto sobresalieron por encima del resto, los cambios fundamentales acaecieron entre 1860 y 1930. En este período se conformó una red jerarquizada de ciudades que abarcaba todo el territorio español. El ritmo de urbanización se aceleró en la Península, afectando a todas las regiones y coincidiendo con un prolongado y ascendente crecimiento demográfico ${ }^{2}$. El número de ciudades de más de 5.000 habitantes pasó de 265 en 1860 a 480 en 1930, y las de más de 10.000 , de 84 a 178 en 1930. El resultado de este proceso desembocó, a principios del siglo XX, en que el 30 por 100 de la población vivía en ciudades mayores de 5.000 habitantes, y que hacia 1930 ya era del 37 por 100. En su conjunto, el ritmo medio de crecimiento de las ciudades estaba acelerándose, pasando de una tasa media del 0,67 anual entre $1860 \mathrm{y}$ 1900, a una tasa del 0,88 entre los años 1900 y 1930.

El nuevo desarrollo urbano exigió la dotación de infraestructuras básicas cuya tecnología fue más accesible a partir de la segunda revolución industrial. Frente a una visión pesimista de las ciudades españolas, existen indicios que permiten vislumbrar una realidad bien diferente. Varios trabajos, desde perspectivas bien distintas, parecen arrojar luz sobre la modernización en las primeras décadas del siglo XX. Este proceso provocó un crecimiento y una diversificación de los servicios urbanos colectivos, así 2017.

${ }_{1}^{1}$ Beascoechea Gangoiti y Otero Carvajal, 2015. Otero-Carvajal y Pallol-Trigueros,

2 Rodríguez Martín, 2015. 
como la necesidad de aumentar las inversiones y recurrir a las empresas privadas mediante concesiones ${ }^{3}$.

Desde el punto de vista de la oferta, el avance de los servicios públicos puede estimarse como parte integrante de la especialización y la división del trabajo. En cuanto a la demanda, es el resultado del cambio de costumbres que trajo el incremento de la renta y la capacidad adquisitiva. Existió una mayor exigencia de servicios a medida que la economía fue mejorando, incluso superando en ocasiones el propio requerimiento de bienes. El crecimiento de los servicios en las ciudades se entiende como un efecto de los otros sectores, especialmente la industria. Dadas las diferencias de productividad, los servicios tienden a crecer proporcionalmente más y a aumentar su parte relativa en el conjunto. En la medida que las ciudades fueron creciendo, los servicios fueron aumentando. La urbanización y la industrialización generaron el incremento de servicios profesionales, que en muy pocas ocasiones se habían planteado en la etapa preindustrial. La demanda fue cubierta por la correspondiente especialización de los funcionarios municipales, las empresas privadas o por otros organismos.

Por otra parte, este incremento de los servicios públicos, coincide con la maltrecha situación económica de los ayuntamientos. La herencia del siglo XVIII dejó un poso de enfrentamiento entre el gobierno central y las entidades locales. El poder central intentaba reforzar su autoridad, pero las ciudades, unas más dinámicas que otras, intentaban llevar a cabo una modernización social y económica. En ese contexto el municipalismo desplegó la bandera de la instalación de los nuevos servicios urbanos. Esta política - signo de modernidad y progreso-, se convirtió en el eje central de los ayuntamientos y los grupos locales como medio para reforzar su poder ${ }^{4}$.

El Estado liberal fue cediendo a los ayuntamientos competencias y responsabilidades relacionadas con los servicios públicos. Por ejemplo, los gobiernos municipales pasaron a ser los responsables de la planificación, organización, coordinación, ejecución y control del abastecimiento de agua ${ }^{5}$. Por estos motivos, optaron por ceder sus prerrogativas en materia de servicios para que interviniera la iniciativa privada. Sin embargo, para no perder el completo control de esas actividades, fueron pergeñando

\footnotetext{
3 Núñez y González, 2008, pp. 373-395. Carreras y Tafunell, 2005.

4 Núñez, 2008.

5 Matés-Barco, 2013.
} 
una complicada y enmarañada red de leyes y decretos. Existía una dicotomía evidente: los ayuntamientos aumentaban sus competencias municipales, pero recurrían a las inversiones privadas para lograr el funcionamiento de los servicios públicos ${ }^{6}$.

A pesar de las dificultades enunciadas las entidades locales intervinieron activamente en la organización de la ciudad. Entre 1870 y 1924, los cabildos españoles se encargaron de gestionar directamente aquellos servicios municipales que estaban al alcance de sus depauperadas haciendas y que no exigían una inversión excesivamente gravosa. Fueron los casos de los cementerios, mataderos y mercados de abastos. En otras ocasiones se trataba de obras que se podían llevar a cabo en varios ejercicios anuales - pavimentación y alcantarillado - o de infraestructuras que se realizaban con el cobro de contribuciones especiales, como fue el caso de los ensanches. Asimismo, se encargaron de gestionar directamente los servicios más relacionados con la salubridad, salud y educación, aunque tuvieron que traspasarlos al Estado con el paso del tiempo. Otros servicios - como el agua, el gas, la electricidad y los tranvías - , tuvieron que cederse en régimen de concesión a las empresas privadas, puesto que exigían grandes inversiones, no se podía fragmentar su ejecución e implicaban una organización industrial y comercial más compleja ${ }^{7}$. En la etapa final del siglo XIX, la revisión de los presupuestos municipales muestra que los ayuntamientos asumieron un gran cúmulo de proyectos y ejecución de obras. Estas actuaciones no sólo se dieron en las grandes ciudades, sino que también se fueron incorporando progresivamente los municipios medianos y pequeños, en servicios como el establecimiento de alumbrado eléctrico, o las conducciones de agua y alcantarillado ${ }^{8}$. Esto significó un aumento de la deuda pública municipal y la necesidad de incrementar el número de técnicos y funcionarios más capacitados. Comenzó a valorarse la especialización profesional y la eficiencia antes que el clientelismo político. Sin embargo, las necesidades generadas por esa masa de población que llegaba a la ciudad implicaba que los recursos financieros, aunque mayores que en épocas anteriores, fueran todavía escasos. Esta situación provocó que los cabildos municipales padecieran una manifiesta incapacidad para afrontar las obras que requería el desarrollo urbano.

\footnotetext{
${ }^{6}$ Núñez, 2013,pp. 919-955.

7 Bartolomé, Fernández-Paradas y Mirás, 2017. Ruiz-Villaverde, 2013.

${ }^{8}$ Heredia-Flores, 2013.
} 
La postura adoptada por los cabildos - sobre la cesión a empresas privadas de algunos servicios básicos - , se ha justificado señalando que en esa época las empresas públicas eran menos eficaces que las privadas, puesto que los organismos públicos carecían de flexibilidad, se encontraban sin capacidad de gestión suficiente y su hacienda no podía afrontar los fuertes desembolsos que eran necesarios. La gama de opciones organizativas fue bastante amplia, pero esencialmente se puede resumir en la gestión directa por parte de los propios ayuntamientos y la cesión de la gestión a empresas privadas mediante la figura administrativa de la concesión. La configuración concreta de tales opciones cambió en cada caso y varió según las ciudades o el momento histórico.

Como ya se ha mencionado, a lo largo del siglo XIX, el Antiguo Régimen había dejado una maltrecha herencia a las haciendas municipales. Esta situación se plasmaba en la diversidad financiera, el fuerte endeudamiento y la tutela interesada del Estado en las Haciendas locales ${ }^{9}$. Las reformas que intentaron los primeros liberales tuvieron escasa relevancia y no llegaron al fondo real del problema. Además, la enajenación del patrimonio municipal, que se inició durante las contiendas contra Francia - Guerra de la Convención (1793-1795), aunque, sobre todo, durante la Guerra de la Independencia (1808-1814) - , continuó en las décadas siguientes y se aceleró en los años de la primera guerra carlista (18331840). Asimismo, los aspectos impositivos de la financiación municipal apenas cambiaron antes de la reforma de 1845, consolidándose la subordinación de las haciendas locales al Estado.

Los grandes municipios solían dedicar buena parte de sus recursos a la financiación de obras públicas y a cubrir las obligaciones derivadas de los intereses y las amortizaciones de las diversas deudas que tenían contraídas. En general, deudas y obras públicas eran las partidas más significativas del gasto municipal especialmente en las grandes ciudades. Es verdad que corrupción y mala gestión administrativa eran la causa de buena parte de esta orientación, pero también en ocasiones era el resultado de innovadores proyectos de modernización de la ciudad.

A su vez, desde 1900 la deuda de los Ayuntamientos se convirtió en una pesada losa que ahogaba a los municipios y que no cesaba de agravar su situación. Los ambiciosos programas de reformas urbanísticas exigían unas inversiones que superaban ampliamente la capacidad económica de

9 Comín, 1996, p. 194. Luxán y Bergasa, 2013,pp. 379-412. 
las entidades locales. Con el paso del tiempo, la despreocupación de los organismos públicos competentes por controlar la prestación del servicio y el régimen de monopolio en el que vivían las empresas privadas perjudicaba en muchas ocasiones a los consumidores. Esta situación degeneró en conflictos con los ayuntamientos que llevó, a partir de 1920, a un mayor control de los concesionarios. Progresivamente, los municipios se vieron más capaces de afrontar la gestión y optaron por recuperar el control de los servicios públicos conforme vencían las concesiones. Varias causas coincidieron para esta nueva situación: finalización del tiempo de la concesión, crisis económica de algunas empresas tras la Primera Guerra Mundial, continua inflación, retraimiento del capital extranjero y, sobre todo, un importante cambio en las posibilidades financieras de los propios ayuntamientos. Más adelante, a partir de la Ley de Régimen Local de 1955, se crearon empresas públicas con una gestión similar a la realizada por las privadas.

En esencia todos apuntaban en la misma dirección: el ahogo de los ayuntamientos era un problema de Hacienda, donde escasos recursos e incapacidad para el crédito se daban la mano mutuamente y generaban una impenetrable maraña que dificultaba la realización de cualquier obra o infraestructura que necesitara cualquier ciudad española. Núñez ha resaltado esta tendencia señalando que los ayuntamientos utilizaban la deuda pública como instrumento de modernización de los servicios públicos. Parece evidente que en las primeras décadas del siglo Xx las entidades locales recurrieron a los inversores privados y a los mercados de capital emergentes, así como a las instituciones financieras que comenzaban a surgir en esos años ${ }^{10}$.

En ese contexto, los artículos recogidos en este dossier pretenden analizar el lugar ocupado por los servicios públicos en la modernización de las ciudades a lo largo de los siglos XIX y XX. Las políticas públicas, junto con las empresas privadas, han jugado un papel preponderante en el diseño de la ciudad.

Una de las notas más características que ha proyectado la ciudad contemporánea ha sido el proceso de «terciarización». Desde mediados del siglo XIX, las zonas urbanas vieron incrementar el peso del sector terciario mediante el desarrollo del comercio y la banca. Asimismo, el desarrollo de los servicios públicos de red - agua, gas, transporte - , trazaron buena

${ }^{10}$ Hernández Marco, 2008. 
parte de la configuración de las ciudades modernas ${ }^{11}$. Este dossier presenta diversos estudios que analizan con detalle algunos casos concretos de ciudades en los siglos XIX y XX.

En primer lugar, Luis Enrique Otero Carvajal y Nuria Rodríguez Martín (Universidad Complutense de Madrid), presentan un trabajo sobre «Infraestructuras urbanas y Modernidad. Madrid, nuevas necesidades y nuevos servicios de una metrópoli moderna, 1900-1936». En sus páginas explican cómo en el último tercio del siglo XIX, el crecimiento demográfico y la expansión urbana agravaron los problemas de hacinamiento y salubridad en las grandes urbes europeas. La nueva sociedad demandó el desarrollo de las infraestructuras y los servicios públicos. Como bien señalan, la ausencia de redes de abastecimiento de agua y de residuos, los insuficientes sistemas de transporte, etc., hubieran provocado un colapso de las ciudades en los albores del siglo Xx. Por tal motivo, en España se aprecia un proceso de modernización en el primer tercio del siglo XX, donde el crecimiento urbano fue acompañado por la mejora de las infraestructuras y los servicios públicos. La gran transformación se llevó a cabo en los años treinta y permitió el acercamiento a los parámetros que detentaban las sociedades europeas modernas.

El segundo estudio aborda «La crisis del alumbrado de gas en las ciudades españolas durante la I Guerra Mundial». La profesora Mercedes Fernández Paradas, de la Universidad de Málaga, analiza las consecuencias que tuvo el conflicto bélico sobre la actividad gasista, especialmente en lo referente al alumbrado. La aguda crisis de esos años se describe con la evolución de la producción de gas, las dificultades empresariales, el número de municipios con iluminación de gas y los ingresos obtenidos por su venta. El detallado recuento de los problemas que padeció la industria del gas - falta de carbón, subida de costes, etc. - , evidencia los duros momentos que padeció la industria del gas. Este análisis se complementa con las estrategias que desarrollaron las empresas de gas para hacer frente a la competencia de la electricidad, las protestas obreras, los incrementos de los costes y la decisión del gobierno de reducir el alumbrado público generado como carbón.

Por su parte, Juan Manuel Matés-Barco (Universidad de Jaén), diserta sobre «El abastecimiento de agua a Barcelona (1850-1939): origen y desarrollo de las compañías privadas». Como su título indica, analiza el pa-

11 Fernández-Paradas, 2009. 
pel que jugaron estas empresas en el proceso de modernización urbana y describe algunas de sus características. En este grupo sobresalió Aguas de Barcelona, que con el paso del tiempo fue adquiriendo buena parte de esas compañías y se convirtió en la dominadora del mercado. Los intentos del ayuntamiento para municipalizar la gestión del servicio fueron vanos y no consiguieron rescatar la concesión. Las razones de esa múltiple aparición de empresas fueron diversas. En primer lugar, la expansión y el Ensanche de la ciudad; y en segundo, las leyes de 1866 y 1879, que ratificaron las competencias municipales e impulsaron las concesiones e empresas privadas. En este sentido, la ciudad de Barcelona es un ejemplo identificativo de este tipo de políticas, que se han mantenido hasta la actualidad.

Los profesores Pedro Amigo Román y Pedro Pablo Ortúñez Goicolea (Universidad de Valladolid), en su estudio sobre «Valladolid y Madrid: carbón, ferrocarril y ciudad (1860-1890)», presentan la evolución urbana de esas dos ciudades, analizada desde la relevancia que pudo jugar el suministro de carbón, recibido a través del ferrocarril en sus respectivas estaciones. Entre 1842 y 1860 el gran crecimiento de la urbe vallisoletana se relaciona con el transporte que precedió al ferroviario del carbón. El Canal de Castilla convirtió la ciudad en un enclave industrial de suma importancia. Por su parte, Madrid continuó siendo una ciudad de servicios en las décadas finales del siglo XIX. En esos años pudo aprovisionarse de carbón a través de las líneas de ferrocarril que detentaban compañías como Norte y MZA. A pesar de todo, las mejoras tarifarias de la primera, no alentaron su desarrollo industrial. Los patrones urbanísticos de las dos ciudades persistieron con el paso del tiempo. Valladolid se reforzó como núcleo industrial y Madrid continuó su crecimiento basándose en los servicios, aunque el propio ferrocarril jugó un papel esencial en su transformación industrial y en la especialización de su fuerza de trabajo.

Por último, Rafael Barquín Gil (UNED) y Miguel Muñoz Rubio (Fundación Ferrocarriles Españoles), han realizado una investigación sobre «Los servicios de cercanías en Madrid y Barcelona: una visión histórica». En su trabajo desarrollan una breve historia comparada del transporte ferroviario de cercanías de las dos grandes ciudades españolas. El ámbito cronológico abarca desde los comienzos de la dictadura del general Franco hasta 2015. Ambos enclaves absorben la mayor parte de estos servicios, pero también las diferencias se asientan en las dispares inversiones realizadas, que han actuado y siguen operando como argumentos de carácter político, que cuestionan el papel de la administración central 
como gestor de las infraestructuras de transporte. En su estudio muestran que Madrid y Barcelona, desde la perspectiva del transporte periurbano, son ciudades con problemas muy disparejos, que surgen de situaciones históricas muy heterogéneas. Por tales motivos, resulta conveniente llevar a cabo un análisis histórico y geográfico, que sobrepase las limitaciones impuestas por la utilización de herramientas de carácter económico. Concluyen su trabajo, resaltando el proceso de convergencia existente en ambas ciudades, respecto a la dotación de servicios de transporte.

Málaga-Jaén, 1 de noviembre de 2018

\section{Bibliografía}

Bartolomé, Isabel, Fernández-Paradas, Mercedes y Mirás Araujo, Jesús (eds.), Globalización, nacionalización y liberalización de la industria del gas en la Europa latina (siglos XIX-XXI), Marcial Pons, Madrid, 2017, pp. 173-192.

Beascoechea Gangoiti, José María y Otero Carvajal, Luis Enrique (eds.), Las nuevas clases medias urbanas. Transformación y cambio social en España, 1900-1936, Catarata, Madrid, 2015.

CARreras, Albert y TAFunell, Xavier (coords.): Estadísticas históricas de España. Siglos XIX y XX, v.I, Fundación BBVA, Bilbao, 2005.

Comín, Francisco, Historia de la Hacienda pública, II. España (1808-1995), Crítica, Barcelona, 1996.

FERNÁNDEZ-PARADAS, Mercedes, «Empresas y servicio de alumbrado público por gas en España (1842-1935)», TST. Transportes, Servicios y Telecomunicaciones, 16, 2009, pp. 108-131.

HEREDIA-FLORES, Víctor Manuel, «Municipalización y modernización del servicio de abastecimiento de agua en España: el caso de Málaga /1860-1930), Agua y Territorio, 1, 2013, p. 103-117. DOI: https://dx.doi.org/10.17561/ at.v1i1.1038

HERNÁNDEZ-MARCO, José Luis, «La banca y la financiación de las infraestructuras urbanas: El Banco de Bilbao antes de la Guerra Civil española», GonZÁLEZ-RUIZ, Luis y MATÉs-B ARCO, Juan Manuel, La modernización económica de los Ayuntamientos: servicios públicos, finanzas y gobiernos municipales, Universidad de Jaén, Jaén, 2008, pp. 313-347.

Luxán Meléndez, Santiago de y Bergasa Perdomo, Óscar, «La Hacienda del Estado liberal», GonZÁLEZ-Enciso, Agustín y Matés-BARCo, Juan Manuel (coords.): Historia Económica de España, Ariel, Barcelona, 2013, pp. 379-412. 
MATÉS-BARCO, Juan Manuel, «La conquista del agua en Europa: los modelos de gestión (siglos XIX y XX)», Agua y Territorio, 1, 2013, pp. 21-29. DOI: https://dx.doi.org/10.17561/at.v1i1.1030

NúÑEZ, Gregorio, «Citadini-Creditori. Municipi e mercati finanziari in Spagna durante il primo terzo del Xx secolo», Storia Urbana, 119, 2008, pp. 101124.

NúÑEZ, Gregorio, «Las ciudades», GonZÁleZ-EnCiso, Agustín y MATÉS-BARCO, Juan Manuel, Historia Económica de España, Ariel, Barcelona, 2013, pp. 919-955.

NúÑEZ, Gregorio y GonZÁLEZ, Luis, «Estado, Ayuntamientos y fuerzas locales: el Banco de Crédito Local de España entre dos dictaduras», GonZÁlEz-RuIZ, Luis y MATÉS-BARCO, Juan Manuel, La modernización económica de los Ayuntamientos: servicios públicos, finanzas y gobiernos municipales, Universidad de Jaén, Jaén, 2008, pp. 373-395.

Otero Carvajal, Luis Enrique y Pallol Trigueros, Rubén (eds.), La sociedad urbana en España, 1900-1936: redes impulsoras de la modernidad, Madrid, Los Libros de La Catarata, pp. 189-228.

Rodríguez MARTín, Nuria, La capital de un sueño. Madrid en el primer tercio del siglo XX, Centro de Estudios Políticos y Constitucionales, Madrid, 2015.

Ruiz-VILLAVERDE, Alberto, «Reflexiones sobre la gestión de los servicios urbanos del agua: Un recorrido histórico del caso español», Agua y Territorio, 1, 2013, pp. 31-40. DOI: https://dx.doi.org/10.17561/at.v1i1.1031 